\title{
A New Proof of Central Limit Theorem for i.i.d. Random Variables
}

\author{
Zhaojun Zong and Feng $\mathrm{Hu}$ \\ School of Mathematical Sciences, Qufu Normal University, Qufu, Shandong 273165, China \\ Correspondence should be addressed to Feng Hu; hufengqf@163.com
}

Received 13 November 2013; Accepted 16 December 2013

Academic Editor: Xinguang Zhang

Copyright (C) 2013 Z. Zong and F. Hu. This is an open access article distributed under the Creative Commons Attribution License, which permits unrestricted use, distribution, and reproduction in any medium, provided the original work is properly cited.

Central limit theorem (CLT) has long and widely been known as a fundamental result in probability theory. In this note, we give a new proof of CLT for independent identically distributed (i.i.d.) random variables. Our main tool is the viscosity solution theory of partial differential equation (PDE).

\section{Introduction}

Central limit theorem (CLT) has long and widely been known as a fundamental result in probability theory. The most familiar method to prove CLT is to use characteristic functions. To a mathematician having been already familiar with Fourier analysis, the characteristic function is a natural tool, but to a student of probability or statistics, confronting a proof of CLT for the first time, it may appear as an ingenious but artificial device. Thus, although knowledge of characteristic functions remains indispensable for the study of general limit theorems, there may be some interest in an alternative way of attacking the basic normal approximation theorem. Indeed, due to the importance of CLT, there exist the numerous proofs of CLT such as Stein's method and Lindeberg's method. Let us mention the contribution of Lindeberg [1] which used Taylor expansions and careful estimates to prove CLT. For more details of the history of CLT and its proofs, we can see Lindeberg [1], Feller [2, 3], Adams [4], Billingsley [5], Dalang [6], Dudley [7], Nourdin and Peccati [8], Ho and Chen [9], and so on.

Recently, motivated by model uncertainties in statistics, finance, and economics, Peng $[10,11]$ initiated the notion of independent identically distributed random variables and the definition of $G$-normal distribution. He further obtained a new CLT under sublinear expectations.

In this note, inspired by the proof of Peng's CLT, we give a new proof of the classical CLT for independent identically distributed (i.i.d.) random variables. Our proof is short and simple since we borrow the viscosity solution theory of partial differential equation (PDE).

\section{Preliminaries}

In this section, we introduce some basic notations, notions, and propositions that are useful in this paper.

Let $C_{b \text {, Lip }}\left(\mathbb{R}^{n}\right)$ denote the class of bounded functions $f$ satisfying

$$
|f(x)-f(y)| \leq C|x-y| \quad \forall x, y \in \mathbb{R}^{n},
$$

for some $C>0$ depending on $f$; let $C\left(\mathbb{R}^{n}\right)$ denote the class of continuous functions $f$; let $C_{b}^{2,3}\left([0, \infty) \times \mathbb{R}^{n}\right)$ denote the class of bounded and 2-time continuously differentiable functions with bounded derivatives of all orders less than or equal to 2 on $[0, \infty)$ and 3 -time continuously differentiable functions with bounded derivatives of all orders less than or equal to 3 on $\mathbb{R}^{n}$.

Let $X$ be a random variable with distribution function $V$, so that, for any $y \in \mathbb{R}$,

$$
\operatorname{Pr}(X \leq y)=V(y) .
$$

If $f$ is any function in $C_{b \text {, Lip }}(\mathbb{R})$, the mathematical expectation of $f(X)$ exists and

$$
E[f(X)]=\int_{-\infty}^{+\infty} f(y) \mathrm{d} V(y) .
$$


Our proof is based on the following classical results for i.i.d. random variables and normally distributed random variables with zero means.

Proposition 1. Suppose $\left\{X_{i}\right\}_{i=1}^{\infty}$ is a sequence of i.i.d. random variables. Then

(i) for each $f \in C(\mathbb{R})$, if $E\left[\left|f\left(X_{1}\right)\right|\right]<\infty$, then $\forall i, j \in \mathbb{N}$,

$$
E\left[f\left(X_{i}\right)\right]=E\left[f\left(X_{j}\right)\right]
$$

(ii) $\forall i \in \mathbb{N}$; for each $f \in C\left(\mathbb{R}^{i+1}\right)$, if $E\left[\left|f\left(Y, X_{i+1}\right)\right|\right]<\infty$, then

$$
E\left[f\left(Y, X_{i+1}\right)\right]=E\left[E\left[f\left(x, X_{i+1}\right)\right]_{Y=x}\right],
$$

where $Y:=\left(X_{1}, \ldots, X_{i}\right)$.

Proposition 2. Suppose $X$ is a normally distributed random variable with $E[X]=0$ and $E\left[X^{2}\right]=\sigma^{2}>0$, denoted by $N\left(0, \sigma^{2}\right)$. Then if $Y \stackrel{d}{=} X$ and $Y$ is independent of $X$, we have, for each $f \in C_{b, L i p}(\mathbb{R})$,

$$
E[f(a X+b Y)]=E\left[f\left(\sqrt{a^{2}+b^{2}} X\right)\right], \quad \forall a, b \geq 0 .
$$

We will show that a normally distributed random variable $X$ with $E[X]=0$ and $E\left[X^{2}\right]=\sigma^{2}>0$ is characterized by the following $P D E$ defined on $[0, \infty) \times \mathbb{R}$ :

$$
\partial_{t} u-\frac{1}{2} \sigma^{2} \partial_{x x}^{2} u=0
$$

with Cauchy condition $u(0, x)=f(x)$. Equation (7) is called the heat equation.

Definition 3. A real-valued continuous function $u \in$ $C([0, \infty) \times \mathbb{R})$ is called a viscosity subsolution (resp., supersolution) for (7), if for each function $v \in C_{b}^{2,3}([0, \infty) \times \mathbb{R})$ and for each minimum (resp., maximum) point $(t, x) \in[0, \infty) \times \mathbb{R}$ of $v-u$, we have

$$
\partial_{t} v-\frac{1}{2} \sigma^{2} \partial_{x x}^{2} v \leq 0 \quad(\text { resp. }, \geq 0)
$$

$u$ is called a viscosity solution for (7) if it is both a viscosity subsolution and a viscosity supersolution.

Remark 4. For more basic definitions, results, and related literature on viscosity solutions of PDEs, the readers can refer to Crandall et al. [12].

Lemma 5. Let $X$ be an $N\left(0, \sigma^{2}\right)$ distributed random variable. For each $f \in C_{b, \text { Lip }}(\mathbb{R})$, we define a function

$$
u(t, x):=E[f(x+\sqrt{t} X)], \quad \forall(t, x) \in[0, \infty) \times \mathbb{R} .
$$

Then we have

$$
u(t+s, x)=E[u(t, x+\sqrt{s} X)], \quad \forall s \geq 0 .
$$

We also have the estimates: for each $T>0$, there exists a constant $C>0$ such that, for all $t, s \in[0, T]$ and $x, y \in \mathbb{R}$,

$$
\begin{gathered}
|u(t, x)-u(t, y)| \leq C|x-y|, \\
|u(t+s, x)-u(t, x)| \leq C s^{1 / 2} .
\end{gathered}
$$

Moreover, $u$ is the unique viscosity solution, continuous in the sense of (11) and (12), of (7) with Cauchy condition $u(0, x)=f(x)$.

Proof. Since

$$
\begin{aligned}
|u(t, x)-u(t, y)| & =|E[f(x+\sqrt{t} X)]-E[f(y+\sqrt{t} X)]| \\
& \leq E[|f(x+\sqrt{t} X)-f(y+\sqrt{t} X)|] \\
& \leq C|x-y|,
\end{aligned}
$$

we then have (11). Let $Y$ be independent of $X$ such that $Y \stackrel{d}{=}$ $X$. By Propositions 1 and 2, we have

$$
\begin{aligned}
u(t+s, x) & =E[f(x+\sqrt{t+s} X)] \\
& =E[f(x+\sqrt{s} X+\sqrt{t} Y)] \\
& =E\left[E[f(x+\sqrt{s} z+\sqrt{t} Y)]_{X=z}\right] \\
& =E[u(t, x+\sqrt{s} X)] .
\end{aligned}
$$

It follows from this and (11) that

$$
\begin{aligned}
|u(t+s, x)-u(t, x)| & =|E[u(t, x+\sqrt{s} X)-u(t, x)]| \\
& \leq E[|u(t, x+\sqrt{s} X)-u(t, x)|] \\
& \leq E\left[C s^{1 / 2}|X|\right],
\end{aligned}
$$

which implies (12).

Now, for a fixed point $(t, x) \in(0, \infty) \times \mathbb{R}$, let $v \in$ $C_{b}^{2,3}([0, \infty) \times \mathbb{R})$ satisfy $v \geq u$ and $v(t, x)=u(t, x)$. By $(10)$, we have, for $\delta \in(0, t)$,

$$
\begin{aligned}
0 \leq & E[v(t-\delta, x+\sqrt{\delta} X)-v(t, x)] \\
\leq & -\partial_{t} v(t, x) \delta+E\left[\partial_{x} v(t, x) \sqrt{\delta} X\right] \\
& +E\left[\frac{1}{2} \partial_{x x}^{2} v(t, x) \delta X^{2}\right]+\bar{C} \delta^{3 / 2} \\
= & -\partial_{t} v(t, x) \delta+\frac{1}{2} \sigma^{2} \partial_{x x}^{2} v(t, x) \delta+\bar{C} \delta^{3 / 2},
\end{aligned}
$$

where $\bar{C}$ is a positive constant, and then, we have

$$
\partial_{t} v(t, x)-\frac{1}{2} \sigma^{2} \partial_{x x}^{2} v(t, x) \leq 0 .
$$

Hence, $u$ is a viscosity subsolution for (7). Similarly, we can prove that $u$ is a viscosity supersolution for (7). The proof of Lemma 5 is completed. 


\section{A New Proof of CLT for i.i.d. Random Variables}

Theorem 6. Let $\left\{X_{i}\right\}_{i=1}^{\infty}$ be a sequence of i.i.d. random variables. We further assume that

$$
\begin{aligned}
& E\left[X_{1}\right]=\mu, \\
& E\left[\left(X_{1}-\mu\right)^{2}\right]=\sigma^{2}>0, \\
& E\left[\left|X_{1}\right|^{3}\right]<\infty .
\end{aligned}
$$

Denote $S_{n}:=\sum_{i=1}^{n}\left(X_{i}-\mu\right)$. Then

$$
\lim _{n \rightarrow \infty} \operatorname{Pr}\left(\frac{S_{n}}{\sqrt{n}} \leq y\right)=\int_{-\infty}^{y} \frac{1}{\sqrt{2 \pi} \sigma} e^{-x^{2} / 2 \sigma^{2}} \mathrm{~d} x .
$$

In order to prove Theorem 6, we need the following lemma.

Lemma 7. Under the assumptions of Theorem 6, we have

$$
\lim _{n \rightarrow \infty} E\left[f\left(\frac{S_{n}}{\sqrt{n}}\right)\right]=E[f(X)]
$$

for any $f \in C_{b, L i p}(\mathbb{R})$, where $X$ is $N\left(0, \sigma^{2}\right)$.

Proof. The main approach of the following proof derives from Peng [10]. For a small but fixed $h>0$, let $v$ be the unique viscosity solution of

$$
\begin{aligned}
& \partial_{t} v+\frac{1}{2} \sigma^{2} \partial_{x x}^{2} v=0, \quad(t, x) \in[0,1+h] \times \mathbb{R}, \\
& v(1+h, x)=f(x) .
\end{aligned}
$$

By Lemma 5,

$$
v(t, x)=E[f(x+\sqrt{1+h-t} X)] .
$$

Particularly,

$$
v(h, 0)=E[f(X)], \quad v(1+h, x)=f(x) .
$$

Since (21) is a uniformly parabolic PDE, thus by the interior regularity of $v$ (see Wang [13]), we have

$$
\|v\|_{C^{1+\alpha / 2,2+\alpha}([0,1] \times \mathbb{R})}<\infty, \quad \text { for some } \alpha \in(0,1) .
$$

We set $\delta:=1 / n$ and $S_{0}:=0$. Then

$$
\begin{aligned}
& v\left(1, \sqrt{\delta} S_{n}\right)-v(0,0) \\
& =\sum_{i=0}^{n-1}\left\{v\left((i+1) \delta, \sqrt{\delta} S_{i+1}\right)-v\left(i \delta, \sqrt{\delta} S_{i}\right)\right\} \\
& =\sum_{i=0}^{n-1}\left\{\left[v\left((i+1) \delta, \sqrt{\delta} S_{i+1}\right)-v\left(i \delta, \sqrt{\delta} S_{i+1}\right)\right]\right. \\
& \left.\quad+\left[v\left(i \delta, \sqrt{\delta} S_{i+1}\right)-v\left(i \delta, \sqrt{\delta} S_{i}\right)\right]\right\} \\
& =\sum_{i=0}^{n-1}\left\{I_{\delta}^{i}+J_{\delta}^{i}\right\} .
\end{aligned}
$$

By Taylor's expansion,

$$
\begin{aligned}
& J_{\delta}^{i}= \partial_{t} v\left(i \delta, \sqrt{\delta} S_{i}\right) \delta+\frac{1}{2} \partial_{x x}^{2} v\left(i \delta, \sqrt{\delta} S_{i}\right)\left(X_{i+1}-\mu\right)^{2} \delta \\
&+\partial_{x} v\left(i \delta, \sqrt{\delta} S_{i}\right)\left(X_{i+1}-\mu\right) \sqrt{\delta}, \\
& I_{\delta}^{i}=\int_{0}^{1}\left[\partial_{t} v\left((i+\beta) \delta, \sqrt{\delta} S_{i+1}\right)-\partial_{t} v\left(i \delta, \sqrt{\delta} S_{i+1}\right)\right] \mathrm{d} \beta \delta \\
&+\left[\partial_{t} v\left(i \delta, \sqrt{\delta} S_{i+1}\right)-\partial_{t} v\left(i \delta, \sqrt{\delta} S_{i}\right)\right] \delta \\
&+\int_{0}^{1}\left[\partial_{x x}^{2} v\left(i \delta, \sqrt{\delta} S_{i}+\gamma \beta \sqrt{\delta}\left(X_{i+1}-\mu\right)\right)\right. \\
&\left.-\partial_{x x}^{2} v\left(i \delta, \sqrt{\delta} S_{i}\right)\right] \gamma \mathrm{d} \beta \mathrm{d} \gamma\left(X_{i+1}-\mu\right)^{2} \delta .
\end{aligned}
$$

Thus

$$
E\left[v\left(1, \sqrt{\delta} S_{n}\right)\right]-v(0,0)=\sum_{i=0}^{n-1} E\left[J_{\delta}^{i}\right]+\sum_{i=0}^{n-1} E\left[I_{\delta}^{i}\right] .
$$

We now prove that

$$
\sum_{i=0}^{n-1} E\left[J_{\delta}^{i}\right]=0
$$

Indeed, for the 3rd term of $J_{\delta}^{i}$, by Proposition 1,

$$
E\left[\partial_{x} v\left(i \delta, \sqrt{\delta} S_{i}\right)\left(X_{i+1}-\mu\right) \sqrt{\delta}\right]=0 .
$$

For the second term of $J_{\delta}^{i}$, by Proposition 1, we have

$$
\begin{gathered}
E\left[\frac{1}{2} \partial_{x x}^{2} v\left(i \delta, \sqrt{\delta} S_{i}\right)\left(X_{i+1}-\mu\right)^{2} \delta\right] \\
=E\left[\frac{1}{2} \sigma^{2} \partial_{x x}^{2} v\left(i \delta, \sqrt{\delta} S_{i}\right) \delta\right] .
\end{gathered}
$$

Thus combining the above two equalities with

$$
\partial_{t} v+\frac{1}{2} \sigma^{2} \partial_{x x}^{2} v=0
$$

we have

$$
\sum_{i=0}^{n-1} E\left[J_{\delta}^{i}\right]=0
$$

Thus, (27) can be rewritten as

$$
E\left[v\left(1, \sqrt{\delta} S_{n}\right)\right]-v(0,0)=\sum_{i=0}^{n-1} E\left[J_{\delta}^{i}\right] .
$$

But since both $\partial_{t} v$ and $\partial_{x x}^{2} v$ are uniformly $\alpha$-hölder continuous in $x$ and $\alpha / 2$-hölder continuous in $t$ on $[0,1] \times \mathbb{R}$, we then have

$$
\left|I_{\delta}^{i}\right| \leq C \delta^{1+\alpha / 2}\left[1+\left|X_{i+1}-\mu\right|^{\alpha}+\left|X_{i+1}-\mu\right|^{2+\alpha}\right] .
$$


Thus

$$
\begin{aligned}
& \left|E\left[v\left(1, \sqrt{\delta} S_{n}\right)\right]-v(0,0)\right| \\
& \quad \leq C\left(\frac{1}{n}\right)^{\alpha / 2}\left(1+E\left[\left|X_{1}-\mu\right|^{\alpha}\right]+E\left[\left|X_{1}-\mu\right|^{2+\alpha}\right]\right),
\end{aligned}
$$

where $C$ is a positive constant. As $n \rightarrow \infty$, we have

$$
\lim _{n \rightarrow \infty} E\left[v\left(1, \sqrt{\delta} S_{n}\right)\right]=v(0,0) .
$$

On the other hand, for each $t, t^{\prime} \in[0,1+h]$ and $x \in \mathbb{R}$,

$$
\left|v(t, x)-v\left(t^{\prime}, x\right)\right| \leq C \sqrt{\left|t-t^{\prime}\right|}
$$

Thus

$$
|v(0,0)-v(h, 0)| \leq C \sqrt{h}
$$

and by (23)

$$
\begin{aligned}
\mid E & {\left[v\left(1, \sqrt{\delta} S_{n}\right)\right]-E\left[f\left(\sqrt{\delta} S_{n}\right)\right] \mid } \\
& =\left|E\left[v\left(1, \sqrt{\delta} S_{n}\right)\right]-E\left[v\left(1+h, \sqrt{\delta} S_{n}\right)\right]\right| \leq C \sqrt{h} .
\end{aligned}
$$

It follows from (23), (36), (38), and (39) that

$$
\limsup _{n \rightarrow \infty}\left|E\left[f\left(\frac{S_{n}}{\sqrt{n}}\right)\right]-E[f(X)]\right| \leq 2 C \sqrt{h} .
$$

Since $h$ can be arbitrarily small, we have

$$
\lim _{n \rightarrow \infty} E\left[f\left(\frac{S_{n}}{\sqrt{n}}\right)\right]=E[f(X)] .
$$

Proof of Theorem 6. For notional simplification, write

$$
\bar{V}(y):=\int_{-\infty}^{y} \frac{1}{\sqrt{2 \pi} \sigma} \mathrm{e}^{-x^{2} / 2 \sigma^{2}} \mathrm{~d} x .
$$

Let $\varepsilon$ be any positive number, and take $\delta$ small enough such that $\bar{V}(y+\delta)-\bar{V}(y-\delta) \leq \varepsilon$. Construct two functions $f, g$ such that

$$
\begin{aligned}
& f(x)=1 \quad \text { for } x \leq y-\delta, \\
& f(x)=0 \quad \text { for } x \geq y, \\
& f(x)=\frac{1}{\delta}(y-x) \quad \text { for } y-\delta<x<y, \\
& g(x)=1 \text { for } x \leq y, \\
& g(x)=0 \text { for } x \geq y+\delta, \\
& g(x)=\frac{1}{\delta}(y-x)+1 \text { for } y<x<y+\delta .
\end{aligned}
$$

Then

$$
\bar{V}(y-\delta) \leq E[f(X)] \leq \bar{V}(y) \leq E[g(X)] \leq \bar{V}(y+\delta),
$$

and for each $n$,

$$
E\left[f\left(\frac{S_{n}}{\sqrt{n}}\right)\right] \leq \operatorname{Pr}\left(\frac{S_{n}}{\sqrt{n}} \leq y\right) \leq E\left[g\left(\frac{S_{n}}{\sqrt{n}}\right)\right] .
$$

Obviously, $f$ and $g \in C_{b \text {, Lip }}(\mathbb{R})$. By Lemma 7, we have

$$
\begin{aligned}
& \lim _{n \rightarrow \infty} E\left[f\left(\frac{S_{n}}{\sqrt{n}}\right)\right]=E[f(X)], \\
& \lim _{n \rightarrow \infty} E\left[g\left(\frac{S_{n}}{\sqrt{n}}\right)\right]=E[g(X)] .
\end{aligned}
$$

So

$$
\begin{aligned}
& E[f(X)] \leq \liminf _{n \rightarrow \infty} \operatorname{Pr}\left(\frac{S_{n}}{\sqrt{n}} \leq y\right) \\
& \quad \leq \limsup _{n \rightarrow \infty} \operatorname{Pr}\left(\frac{S_{n}}{\sqrt{n}} \leq y\right) \\
& \leq E[g(X)] .
\end{aligned}
$$

Hence

$$
\begin{aligned}
& \bar{V}(y)-\varepsilon \leq \liminf _{n \rightarrow \infty} \operatorname{Pr}\left(\frac{S_{n}}{\sqrt{n}} \leq y\right) \\
& \quad \leq \limsup _{n \rightarrow \infty} \operatorname{Pr}\left(\frac{S_{n}}{\sqrt{n}} \leq y\right) \\
& \leq \bar{V}(y)+\varepsilon .
\end{aligned}
$$

Since this is true for every $\varepsilon$, we have

$$
\lim _{n \rightarrow \infty} \operatorname{Pr}\left(\frac{S_{n}}{\sqrt{n}} \leq y\right)=\bar{V}(y)
$$

\section{Conflict of Interests}

The authors declare that there is no conflict of interests regarding the publication of this paper.

\section{Acknowledgments}

The authors would like to thank the editor and the anonymous referees for their careful reading of this paper, correction of errors, and valuable suggestions. The authors thank the partial support from the National Natural Science Foundation of China (Grant nos. 11301295 and 11171179), the Doctoral Program Foundation of Ministry of Education of China (Grant nos. 20123705120005 and 20133705110002), the Postdoctoral Science Foundation of China (Grant no. 2012M521301), the Natural Science Foundation of Shandong Province of China (Grant nos. ZR2012AQ009 and ZR2013AQ021), and the Program for Scientific Research Innovation Team in Colleges and Universities of Shandong Province of China.

\section{References}

[1] J. Lindeberg, "Eine neue Herleitung des Exponentialgesetzes in der Wahrscheinlichkeitsrechnung," Mathematische Zeitschrift, vol. 15, no. 1, pp. 211-225, 1922. 
[2] W. Feller, An Introduction to Probability Theory and Its Applications, vol. 2 of 2 nd edition, John Wiley \& Sons, New York, NY, USA, 1971.

[3] W. Feller, "The fundamental limit theorems in probability," Bulletin of the American Mathematical Society, vol. 51, no. 11, pp. 800-832, 1945.

[4] W. J. Adams, The Life and Times of the Central Limit Theorem, vol. 35 of History of Mathematics, Kaedmon Publishing, New York, NY, USA, 2nd edition, 2009.

[5] P. Billingsley, Probability and Measure, John Wiley \& Sons, New York, NY, USA, 3rd edition, 1995.

[6] R. C. Dalang, "Une démonstrationélémentaire du théorème central limite," Elemente der Mathematik, vol. 60, no. 1, pp. 1-9, 2005.

[7] R. M. Dudley, Real Analysis and Probability, Cambridge University Press, New York, NY, USA, 2nd edition, 2002.

[8] I. Nourdin and G. Peccati, Normal Approximations with Malliavin Calculus, vol. 192 of From Stein's Method to Universality, Cambridge University Press, New York, NY, USA, 2012.

[9] S. T. Ho and L. H. Y. Chen, "An $L^{p}$ bound for the remainder in a combinatorial central limit theorem," Annals of Probability, vol. 6, no. 2, pp. 231-249, 1978.

[10] S. G. Peng, "Law of large numbers and central limit theorem under nonlinear expectations," http://arxiv.org/abs/math/0702358.

[11] S. G. Peng, "A new central limit theorem under sublinear expectations," http://arxiv.org/abs/0803.2656 .

[12] M. G. Crandall, H. Ishii, and P. L. Lions, "User's guide to viscosity solutions of second order partial differential equations," Bulletin of the American Mathematical Society, vol. 27, no. 1, pp. $1-67,1992$.

[13] L. H. Wang, "On the regularity theory of fully nonlinear parabolic equations: II," Communications on Pure and Applied Mathematics, vol. 45, no. 2, pp. 141-178, 1992. 


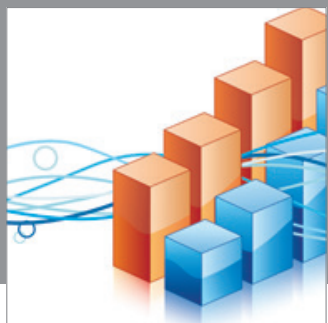

Advances in

Operations Research

mansans

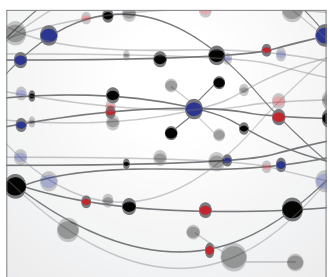

The Scientific World Journal
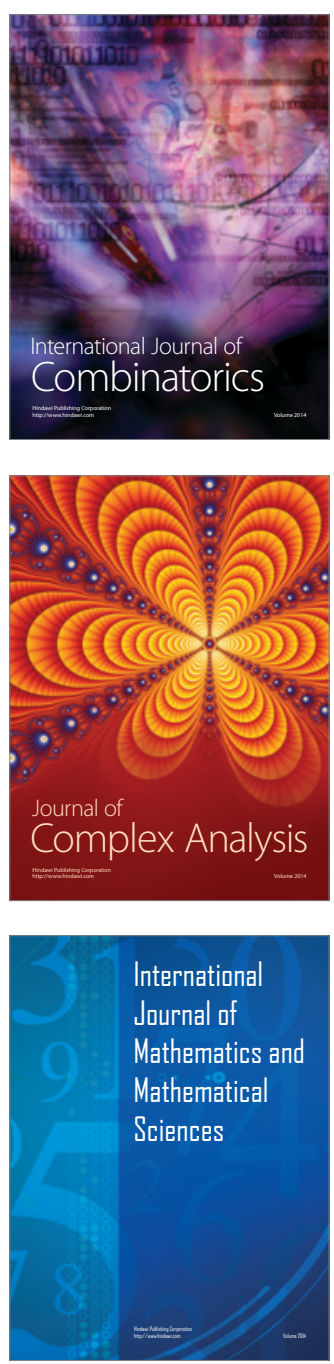
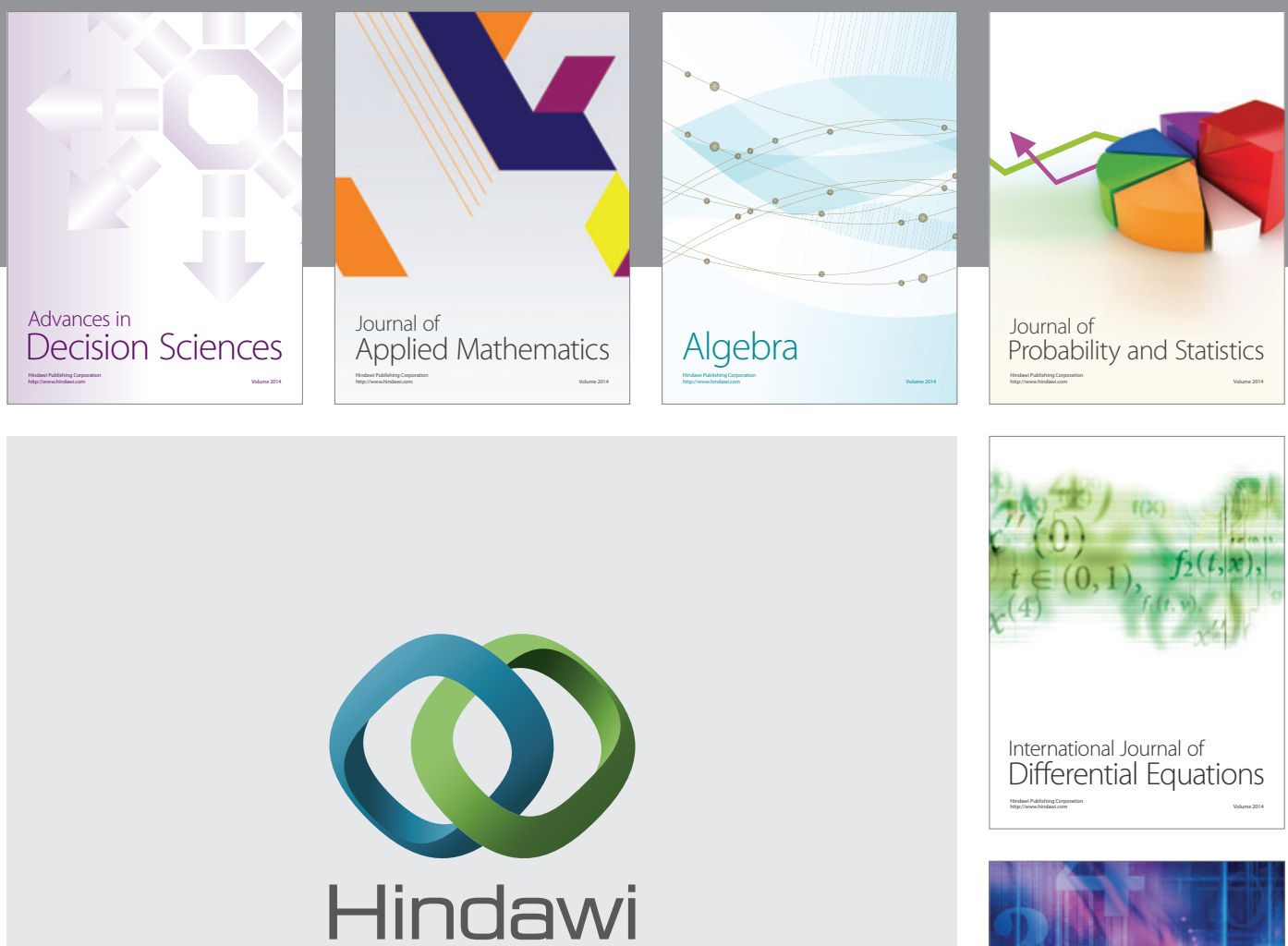

Submit your manuscripts at http://www.hindawi.com
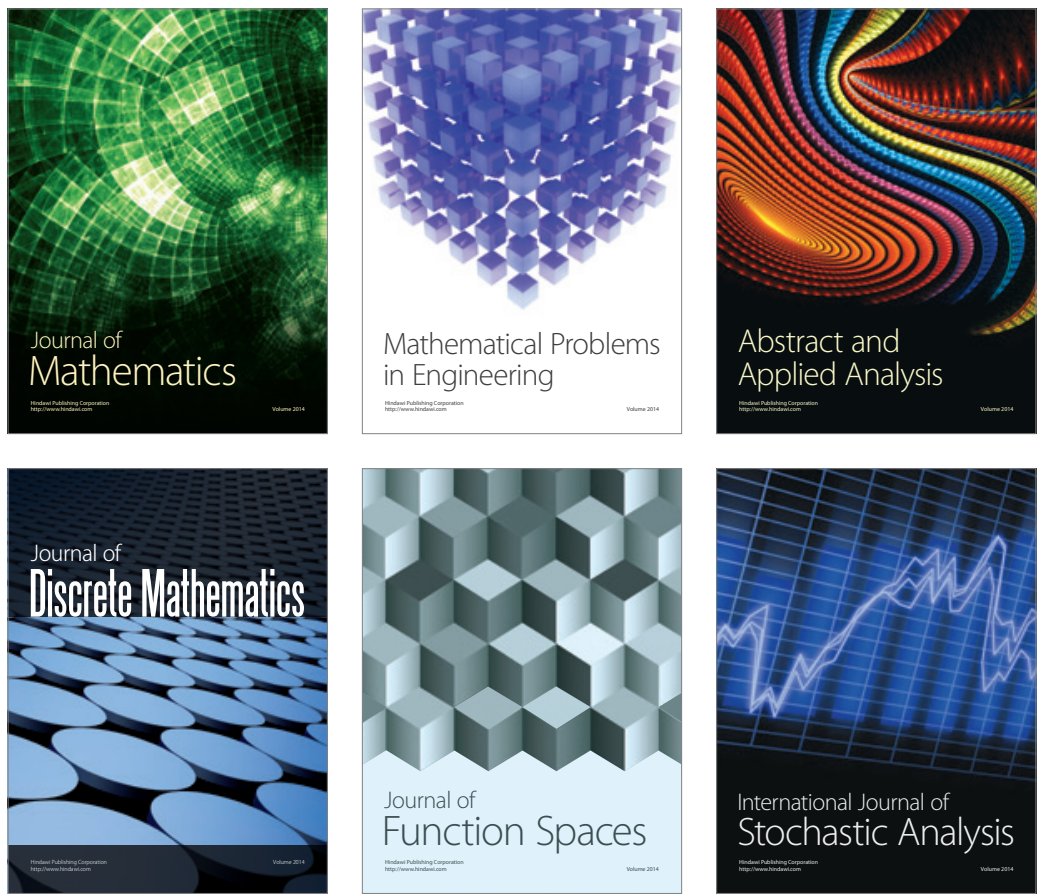

Journal of

Function Spaces

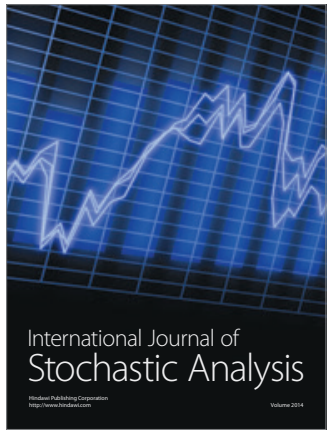

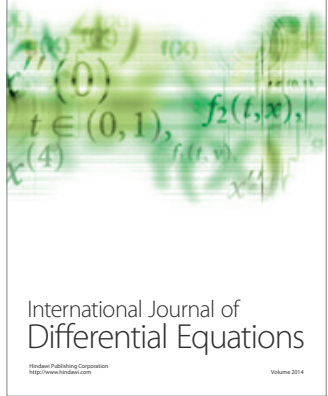
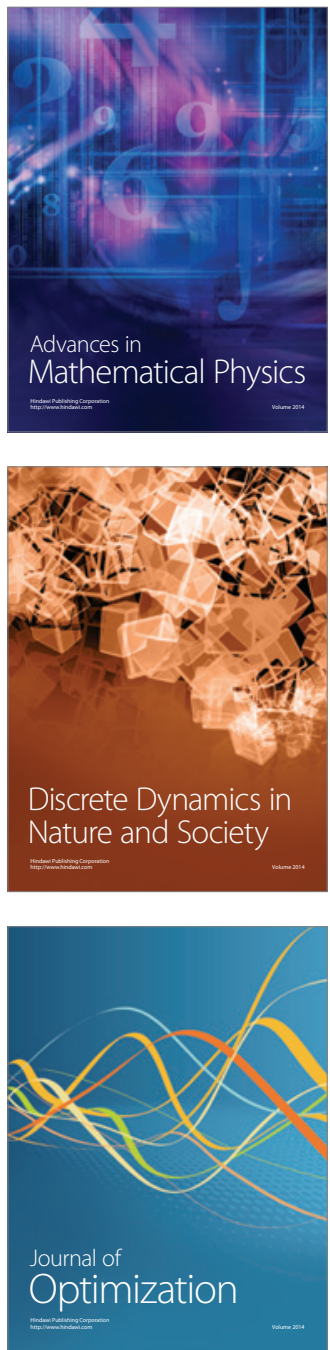